\title{
Supernovae and the Cosmic Distance Scale
}

\author{
By ROBERT P. KIRSHNER
}

Harvard-Smithsonian Center for Astrophysics

60 Garden St., Cambridge, MA 02138, USA

\begin{abstract}
Knowledge of the size and age of the Universe depends on understanding supernovae. The direct geometric measurement of the circumstellar ring of SN 1987A using IUE spectra and HST images provides an independent test of the Cepheid distance scale to the Large Magellanic Cloud. Understanding the details of the mass distribution in the circumstellar matter is important to improving the precision of this distance. Type Ia supernovae have a narrow distribution in absolute magnitude, and new Cepheid distances to IC 4182 (the site of SN 1937C) and to NGC 5253 (the site of SN 1972E) obtained with HST by Sandage and his collaborators allow that absolute magnitude to be calibrated. Comparison with more distant SN Ia gives $H_{0}=$ $56 \pm 8 \mathrm{~km} \mathrm{~s}^{-1} \mathrm{Mpc}^{-1}$. Recent work in supernova spectroscopy and photometry shows that the apparent homogeneity of $\mathrm{SN} \mathrm{Ia}$ is not quite what it seems, and a deeper understanding of these variations is needed to use the SN Ia to best advantage. The Expanding Photosphere Method (EPM) allows direct measurement to each Type II supernova that has adequate photometry and spectroscopy. There are now 18 such objects. The sample of EPM distances from $4.5 \mathrm{Mpc}$ to $180 \mathrm{Mpc}$ indicates $H_{0}=73 \pm 6$ (statistical) \pm 7 (systematic) $\mathrm{km} \mathrm{s}^{-1} \mathrm{Mpc}^{-1}$. Better understanding of supernova atmospheres can reduce the systematic error in this approach, which is completely independent of all other astronomical distances.
\end{abstract}

\section{Introduction}

Measuring the Hubble Constant is one of the goals of observational cosmology. Supernova observations provide powerful and diverse ways to approach this problem. In recent years, work done on Planetary Nebula Luminosity Functions, Surface Brightness Fluctuations, and the Line-Width Luminosity Relation has reached an apparent consensus on the value of the Hubble constant, $H_{0}=80 \pm 10 \mathrm{~km} \mathrm{~s}^{-1} \mathrm{Mpc}^{-1}$ (Jacoby et al. 1992). Yet, good arguments for a "long" distance scale have been advanced by Sandage et al. (1992, 1994) using SNe Ia as standard candles. If $H_{0}$ really is $80 \mathrm{~km} \mathrm{~s}^{-1} \mathrm{Mpc}^{-1}$, and $\Omega_{0}=1$ as preferred by many, then the 8 billion year expansion age of the Universe implied by $H_{0}$ is substantially shorter than the lifetime of the oldest stars (Renzini 1991). Since there is disagreement among reliable workers, and our overall picture for cosmic evolution is at stake, it is useful to evaluate the distance scale using different approaches. Supernova 1987A helps to check the Cepheid scale, SN Ia form the basis for the standard candle approach, and the Expanding Photosphere Method applied to SN II provides independent distances that are now sufficiently numerous and accurate to be compared with other estimates of extragalactic distances.

\section{The Ring Around SN 1987A and the Distance to the LMC}

Supernova 1987A was assiduously studied with the IUE satellite (Pun et al. 1984), and one of the most interesting results was the detection of narrow emission lines in the UV, where emission from the supernova debris was very faint. These lines appeared after about 80 days, and grew in strength for about 400 days. Fransson et al. (1989) developed an interpretation of this emission, attributing it to fluorescence from a shell of material excited by the initial pulse of UV emission produced by the arrival of the shock 


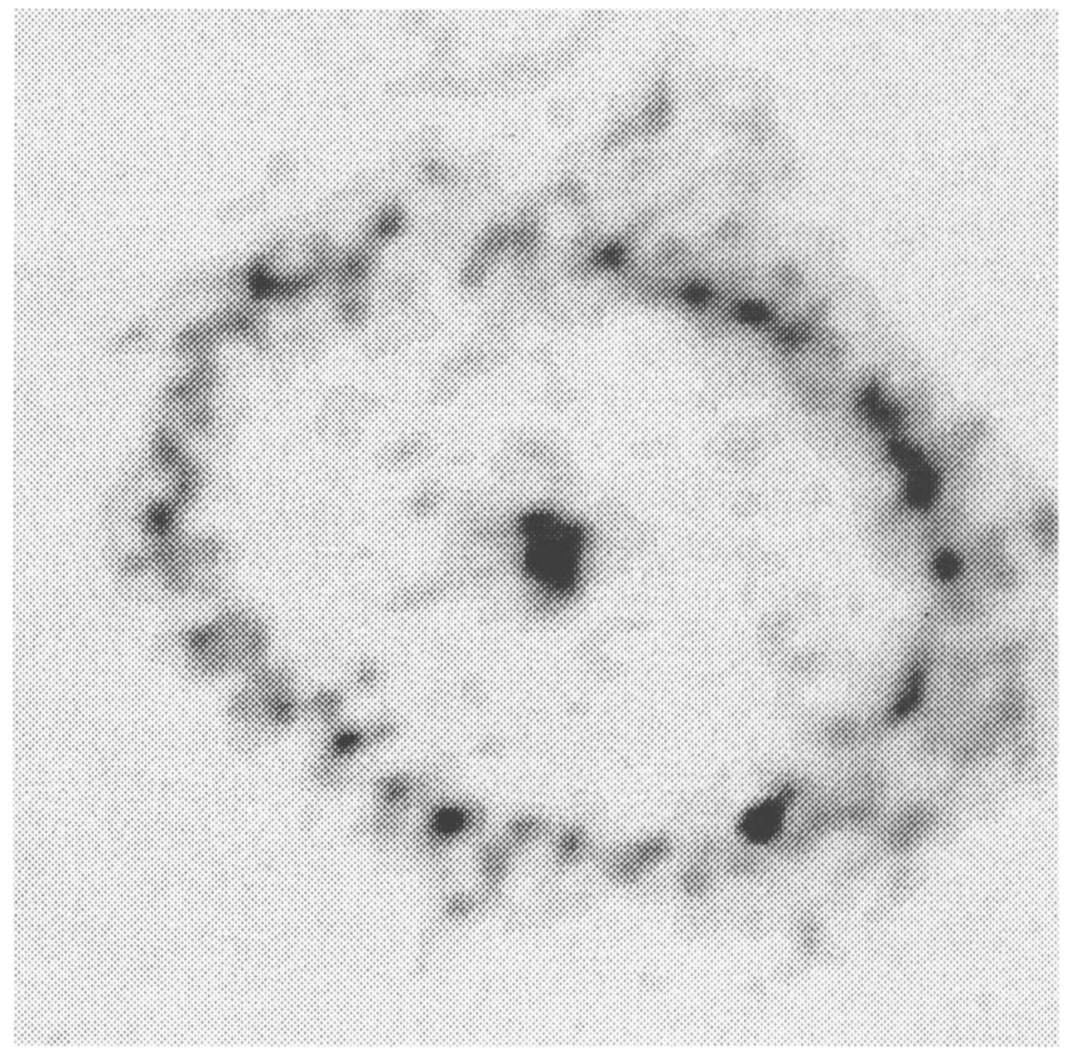

FIGURE 1. An image of the circumstellar ring around SN 1987A taken in the light of [O III] with the Faint Object Camera aboard the Hubble Space Telescope in May 1993.

wave at the surface of the B3 III star. The shell was inferred to lie at a distance of about 200 light days, half the time it took for the exciting pulse to reach the shell and then to reach the observer. In this picture, the emitting gas was a dense shell at the interface between the blue supergiant's wind and the earlier mass loss from the star when it was a red supergiant.

Ground-based observations, and observations with the Faint Object Camera on the Hubble Space Telescope showed that this picture was generally correct, but the details were even more interesting, as shown in Figure 1. Fransson et al. were parsimonious with their hypotheses: given a radius, they inferred a sphere. However, nature was more creative, and the surface brightness distribution of the emission indicates that the fluorescent material is more like a torus (Jakobsen et al. 1991). The elliptical appearance is most likely the projection of a circular ring, seen inclined at an angle of about 43 degrees.

Since the physical size of the ring can be inferred from the time delay as $1.27 \pm 0.0710^{18}$ $\mathrm{cm}$ and the angular size of the major axis is $1.66 \pm 0.03$ ", this gives the distance directly as $51.2 \pm 3.1 \mathrm{kpc}$. This is consistent with the distance to the LMC based on Cepheids (Walker, 1987). Felten (in this conference) has considered a number of systematic effects that might alter the distance and its error estimate, and has drawn attention to the difference between the predicted and the observed time history of the fluorescent emission. 
Gould (1993) has also examined this distance estimate and McCall (1993) has emphasized the location of SN 1987A in the LMC.

A complete understanding of the observed narrow-line emission and its time history is not yet in hand, but the HST data we have obtained from 1990 through 1993 will help. An analysis by Plait et al. (1994) of these FOC images helps determine the geometry of the ring and the inhomogeneity of the gas distribution.

A second geometric distance to the LMC will result from future HST measurements. We know the expansion velocity of the supernova debris and we are just beginning to measure the angular expansion from our FOC images. If the supernova debris is not too asymmetric, this should yield another direct determination of the distance to SN 1987A.

\section{HST Calibration of SN Ia: How Standard the Candles?}

Type Ia supernovae form a more-or-less homogeneous class of objects which shows a moderately narrow distribution in absolute magnitude (Branch and Miller 1993). This observational fact is plausible because theory suggests that $\mathrm{SN}$ Ia arise from the sudden thermonuclear burning of a carbon-oxygen white dwarf at the Chandrasekhar limit. In that case, the Type Ia event has a well-defined mass and composition and the resulting luminosity would be similar from one event to the next. If the luminosity of a single SN Ia could be determined from a measured distance and apparent magnitude, then the observed Hubble diagram for SN Ia could be used to determine the Hubble Constant.

A novel approach to this problem has been explored by Kirshner et al. (1987) and by Smith et al. (1991) who have used observations of the remnants of galactic supernovae to determine their distances by comparing observed proper motions with the shock velocity determined from a theory of non-radiative shocks. While this produces distances to SN 1006 and to Tycho's supernova of 1572, the photometry ("dazzling in brightness") is not sufficiently precise to serve as the fiducial point for cosmic distances.

A more conventional approach has been adopted by Sandage and his collaborators (1992, 1994) using the Hubble Space Telescope. They have used HST to search for Cepheid variables in galaxies which have had well-observed SN Ia. In particular, they have surveyed IC 4182, the site of SN 1937C, and NGC 5253, the site of SN 1972E and of SN 1895. The advantages of HST, even with spherical aberration, are that faint stars in crowded fields can be resolved and that the observing schedule is not subject to the same constraints that affect attempts to detect periodic sources from terrestrial sites. In IC 4182, the observations produced 27 Cepheids with periods ranging from 2 days to over 50 days, and in NGC 5253, there are 12 Cepheids with periods on the range from 4 to 16 days. By comparing the apparent magnitudes of these Cepheids with the local periodluminosity relation, Sandage et al. infer the distance to the galaxies and the absolute magnitude of SN Ia to be $M_{V}=-19.5$. Using this value of the absolute magnitude to calibrate the Hubble diagram for SN Ia (Sandage and Tammann 1993), yields a value of the Hubble constant: $H_{0}=56 \pm 8 \mathrm{~km} \mathrm{~s}^{-1} \mathrm{Mpc}^{-1}$.

This interesting result should be subjected to careful examination. Details of the Cepheid photometry and assumptions about reddening seem reasonable, but should be examined by people who are knowledgeable in those areas. The assumptions about supernovae point to interesting areas for future work. There is good evidence for the photometric and spectroscopic variation of SN Ia as reported by Phillips (1993) and by Suntzeff (this volume). In particular, SN 1991bg (Leibundgut et al. 1993) and SN 1991T (Phillips et al. 1992) appear to be examples SN Ia which are far from typical. This raises the question of whether there are intermediate types of slightly brighter and slightly fainter SN Ia which are lumped together in the usual Hubble diagram for SN Ia. Specif- 
ically, it will be interesting to see whether SN 1937C or SN 1972E shares, to a lesser degree, the features of these not-quite-typical supernovae. In any case, it is essential to pursue the observation and analysis of SN Ia with modern detectors since good light curves and spectra will be essential to sorting out the connection between observable distance-independent supernova properties and supernova luminosities.

A correlation between, for example, the rate of decline after maximum light and the luminosity of a supernova is not all bad. If we have good data in hand and we develop an understanding of photometric and spectroscopic clues to the intrinsic luminosity of the event, we can use that information to reduce the scatter in the Hubble diagram and to improve the estimate of $H_{0}$ based on SN Ia. While a certain amount of skepticism toward any particular value of $H_{0}$ and an understanding that the errors may be larger than quoted is healthy, the deeper lesson may be that the mechanism of SN Ia events is not as thoroughly understood as we might like. In the case of SN $1991 \mathrm{bg}$, Ruiz-Lapuente et al. (1993) have shown that the explosion of a star well below the Chandrasekhar limit may be the best explanation for the observations. If that is really correct, then theoretical homogeneity is too simple and should be replaced with an exploration of the theoretical possibilities as carried out by Höflich et al. (this volume).

\section{The Expanding Photosphere Method: Custom Yardstick for the Cosmos}

Kirshner \& Kwan (1974) exploited a suggestion by Leonard Searle to use SNe II to measure extragalactic distances in a way that is completely independent of every other step in the cosmic distance ladder. This technique, the Expanding Photosphere Method (EPM), was applied to two nearby SNe II and produced distances consistent with $H_{0}=60 \mathrm{~km} \mathrm{~s}^{-1} \mathrm{Mpc}^{-1}$. This work, which assumed SNe II emit like perfect blackbodies, was extended by Wagoner (1977), who demonstrated EPM could, in principle, be applied to SNe II at large redshifts to measure $q_{0}$. Branch et al. (1983) and Kirshner (1985) applied EPM to additional SNe II, but the limitations of these primitive results of EPM were highlighted by Wagoner (1981), who noted that SNe II have scattering-dominated atmospheres, and therefore radiate with a smaller surface flux than a blackbody with the same color temperature. EPM was lifted to solid theoretical and empirical ground after several groups produced sophisticated NLTE atmospheric models of SN 1987A (Eastman \& Kirshner 1989; Höflich 1988; Schmutz et al. 1990; Chilkuri and Wagoner 1988) that gave distances to the LMC which agreed with those derived using Cepheid variable stars (Walker 1987) and with the geometric measurement described above. Schmidt, Kirshner \& Eastman (1992) [SKE92] exploited the tools developed for SN 1987A to measure the distances to ten SNe II, and found these distances consistent with $H_{0}=60 \pm 10 \mathrm{~km} \mathrm{~s}^{-1} \mathrm{Mpc}^{-1}$. Eastman, Schmidt \& Kirshner (1994) [ESK94], have improved these distances using a large grid of models which are not specific to SN 1987A and its compact progenitor. The relation between color temperature and flux inferred from these models generally produces distances $10 \%$ smaller than those based on the cruder formulation by SKE92. The distance measurements reported here use these improved models. Recently, Schmidt et al. (1994a) demonstrated the range over which EPM could be applied by developing the technique for applying EPM on SNe II at large redshifts, and measuring the distance to SN 1992am at a redshift of $c z=14600 \mathrm{~km} \mathrm{~s}^{-1}$. Additional EPM distances to five SNe II observed at Cerro Tololo Inter-American Observatory (CTIO) as part of the Calan/Tololo SN search and monitoring program being carried out as a collaborative effort between the University of Chile and CTIO (Hamuy et al. 1993) have been determined by Schmidt et al. (1994b). When combined with 
previous studies, the new data brings the number of EPM distances to 18. These distances now extend from the LMC $(D=49 \mathrm{kpc})$ to $\mathrm{SN} 1992 \mathrm{am}(D=180 \mathrm{Mpc})-$ an unparalleled range of distances for an extragalactic distance indicator. The host galaxies of these SNe II are typically spirals which, if not too distant or face-on, have distances which can be measured using the Line-Width Luminosity Relation (Tully and Fisher 1977). We compare EPM and Tully-Fisher distances to 11 galaxies which have distances measured both ways.

EPM has been discussed in detail by SKE92. They show that the photospheric angular size of a SNe II is given, for $z<<1$, by

$$
\theta=\frac{R}{D}=\sqrt{\frac{f_{\lambda}}{\zeta_{\lambda}^{2} \pi B_{\lambda}(T)}},
$$

where $T$ is the SN's observed color temperature, $f_{\lambda}$ is the observed flux density, $B_{\lambda}(T)$ is the Planck function evaluated at $T$, and $\zeta_{\lambda}$ is a distance correction factor derived from model atmospheres to account for the dilution effects of scattering atmospheres. SNe II expand freely at velocity, $v$ (measured from the absorption minima of optically thin lines such as Fe II 5169), so that a supernova's photospheric radius, $R$, at any time, $t$, is

$$
R=v\left(t-t_{0}\right)+R_{0}
$$

The initial radius, $R_{0}$, is negligible at all but the earliest epochs, and combining equations (1) and (2) yields

$$
t=D\left(\frac{\theta}{v}\right)+t_{0}
$$

Given at least two measurements of $t, \theta$, and $v$, determined from a calendar, photometry, and spectra, it is possible to solve for both the distance to the $\mathrm{SN}, D$, and the time of explosion, $t_{0}$, simultaneously. It is also possible to test the performance of the method through equation (3) if several measurements of $t, v$, and $\theta$ are available; despite large changes in $T, v, R$, and $\theta$, the distance $D$ should remain constant.

SKE92 demonstrated that the distance correction factors, $\zeta_{\lambda}$, as derived empirically, varied only as a function of color temperature, and not from supernova to supernova. ESK94 have investigated the effects of metallicity, progenitor mass, luminosity, density, and physical structure on the distance correction factor with a grid of 88 models, and found that $\zeta_{\lambda}(T)$ is not sensitive to details of the progenitor star. However, the new distance correction factors of ESK94 give 10\% smaller distances on average than those determined from the models of SKE92.

SKE92 relied on the distance correction factors derived from SN 1987A's VI photometry in their formulation of $\zeta_{\lambda}(T)$. These distance correction factors were applied to objects for which only $B V$ photometry was available. Although the values of $\zeta_{\lambda}(T)$ determined from $B V$ and $V I$ are similar, they are not identical, and this small difference is responsible for most of the systematic difference in the distances derived. In addition, SN 1987A, which resulted from the explosion of a compact progenitor, had a slightly different behavior of $\zeta_{\lambda}(T)$ than typical SNe II with larger initial radii. The custom-crafted models created for SN 1987A (Eastman \& Kirshner 1989) are not quite in the center of the distribution of models, which cover a wide range of plausible initial conditions, computed by ESK94. The distance correction factors determined from models by ESK94 are superior to those of SKE92, and we adopt their formulation of $\zeta_{\lambda}(T)$.

$\mathrm{SNe}$ II occur in galaxies with recent star formation, and the effects of extinction cannot be ignored. SKE92 demonstrated that many SNe II have uniform color evolution during their photospheric phases ( 100 days following explosion), and it is possible to estimate 
the color excess, $E(B-V)$, with a scatter of 0.1 mag. Furthermore, the distances derived using EPM are affected by both attenuation and reddening of the light which cancel to a large extent. The uncertainty that extinction produces in a distance is usually less than $10 \%$. In a worst case example, the distance to SN 1973R, which had more than 2.5 magnitudes of visual extinction, has a distance uncertainty due to extinction of about 20\% (SKE92). EPM distances are not uniformly increased or decreased by extinction, but instead depend on the evolution of the SN and the spacing of the observations. Errors in the reddening are unlikely to bias the average result even when they add noise to individual distances.

We have employed both Monte Carlo and bootstrapping techniques to derive realistic errors for our distance measurements. These account very well for the measuring errors, but some of the uncertainty in EPM arises from systematic differences between the derived and actual values of parameters such as $v$, and $\zeta_{\lambda}$. Except in pathological cases, these errors will cause $\theta / v$ vs. $t$ to deviate from a straight line - if EPM is suffering severe systematic problems, they will be apparent. However, small systematic shifts, which are not time dependent, will not be so obvious, and require a more thoughtful analysis. Several groups have estimated the distance to SN 1987A using different models. The spread in distances suggests that EPM has systematic errors less than $\pm 10 \%$. Aside from the measuring accuracy of the photometry and spectra, the precision of EPM depends on the age of the SN at the time of the first observations and the spacing of subsequent observations. Observations which begin within three weeks of the explosion date, and which continue on a regular basis (once per week) afterwards constrain the time of explosion and reduce the uncertainty in a derived distance. When we have a set of 5 or more observations, spaced a week apart, and of good quality (e.g. $5 \%$ photometry, absorption features clearly visible), the statistical error is smaller than the systematic uncertainties in EPM. In these cases, increasing the precision of the photometry and quality of the spectra, or increasing the observing frequency, will not improve the accuracy of the derived distances. Some of our recent observations have sufficiently good data that the estimated error in the distance approaches our uncertainty in the systematics of the method.

Some of the host galaxies for these SNe II are close enough that EPM can be compared to distances measured using conventional techniques such as Cepheids, while others are at twice the distance to Coma, so that perturbations in the Hubble flow should be small. In between, we can make a galaxy-by-galaxy comparison with Tully-Fisher distances. Three galaxies have Cepheid and EPM distances in common, the LMC (SN 1987A), M 81 (SN 1993J), and M 101 (SN 1970G). In the case of the LMC and M 101, the distances derived by the Cepheids, $49 \pm 4 \mathrm{kpc}$ (Walker 1987) and 7.1 $\pm 0.3 \mathrm{Mpc}$ (Cook, Aaronson, and Illingworth 1986) respectively, are indistinguishable from those measured with EPM, $49 \pm 6 \mathrm{kpc}$ (Eastman \& Kirshner 1989) and 7.4 $4_{-1.5}^{+1.0} \mathrm{Mpc}$ (ESK94). However, EPM appears to give a distance to $\mathrm{M} 81,2.6 \pm 0.4 \mathrm{Mpc}$ (Schmidt et al. 1993), which is $25 \%$ smaller than that derived using Cepheids, 3.6 $\pm 0.4 \mathrm{Mpc}$ (Freedman et al. 1994). It should be noted that SN 1993J has an atypical spectroscopic and photometric evolution which suggests the progenitor lost most of its hydrogen envelope before exploding. These observations, combined with evidence for asymmetry (Januzzi et al. 1993), suggest applying EPM to this object demands extreme care and models specific to the event. Baron et al. (1993) have specifically modeled SN 1993J, and derive a distance to the SN in perfect agreement with the Cepheid distance. However, the larger distance derived by this group may result from them placing the material which forms the photosphere at a higher velocity than that inferred from the Fe II $\lambda \lambda 5169,5018$ absorption lines by Schmidt et al. (1993). A detailed comparison of the two sets of models is needed to sort out this discrepancy. 


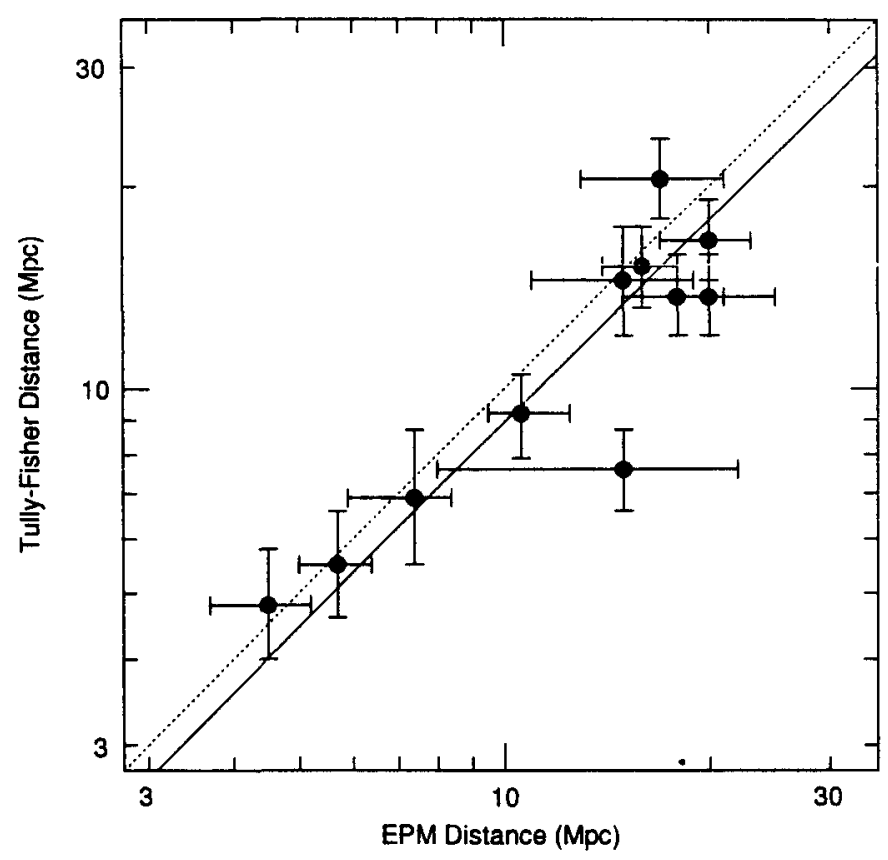

FIGURE 2. Comparison of distances for supernovae with EPM measurements and Tully-Fisher distances to the parent galaxies.

Two other SNe II, SN 1979C and SN 1986L, also have peculiar spectroscopic features (although not to the extent of SN 1993J).

Eleven galaxies have both EPM and Tully-Fisher (TF) distances. Following Pierce (1994), we plot the distances against each other in Figure 2, and find good correlation. However the TF distances average $11 \% \pm 7 \%$ smaller than those determined using EPM. This difference, which is marginally significant, is not surprising given that both TullyFisher or EPM may have systematic errors approaching $10 \%$ due to calibration (in the case of TF) and uncertainties in the physics of SNe II (EPM). More interestingly, the slope is unity, within the estimated uncertainties. This means there is no systematic difference between the distances derived by T-F and EPM nearby and far away. Because EPM does not make any standard candle assumption (and is therefore immune to the usual Malmquist Bias problems), the sample of T-F distances here also has no significant distance-dependent error. This conclusion differs from the view presented by Sandage (1994), who attributes the large value of the Hubble Constant obtained through LineWidth Luminosity Relations to Malmquist Bias.

The observed large scale perturbations in the Hubble flow (Aaronson et al. 1982; Lynden-Bell et al. 1988; Mathewson et al. 1992; Lauer and Postman 1993) make measuring $H_{0}$ from nearby galaxies a risky proposition. The Virgo cluster significantly affects the local Hubble flow (Aaronson et al. 1982), and most of our EPM sample have distances comparable or smaller than the distance to the Virgo Cluster. After correcting the observed heliocentric redshifts for each galaxy for Galactic rotation $\left(220 \mathrm{~km} \mathrm{~s}^{-1}\right)$, we apply a non-linear infall model (Schechter 1980; SKE92) to the sample using a range of parameters discussed by Huchra (1988). Except for the two SNe which lie in the Virgo Cluster, SN 1979C and SN 1988A, the choice of these parameters does not strongly affect the derived recession velocities. SN 1987A and SN 1993J have redshifts which are 


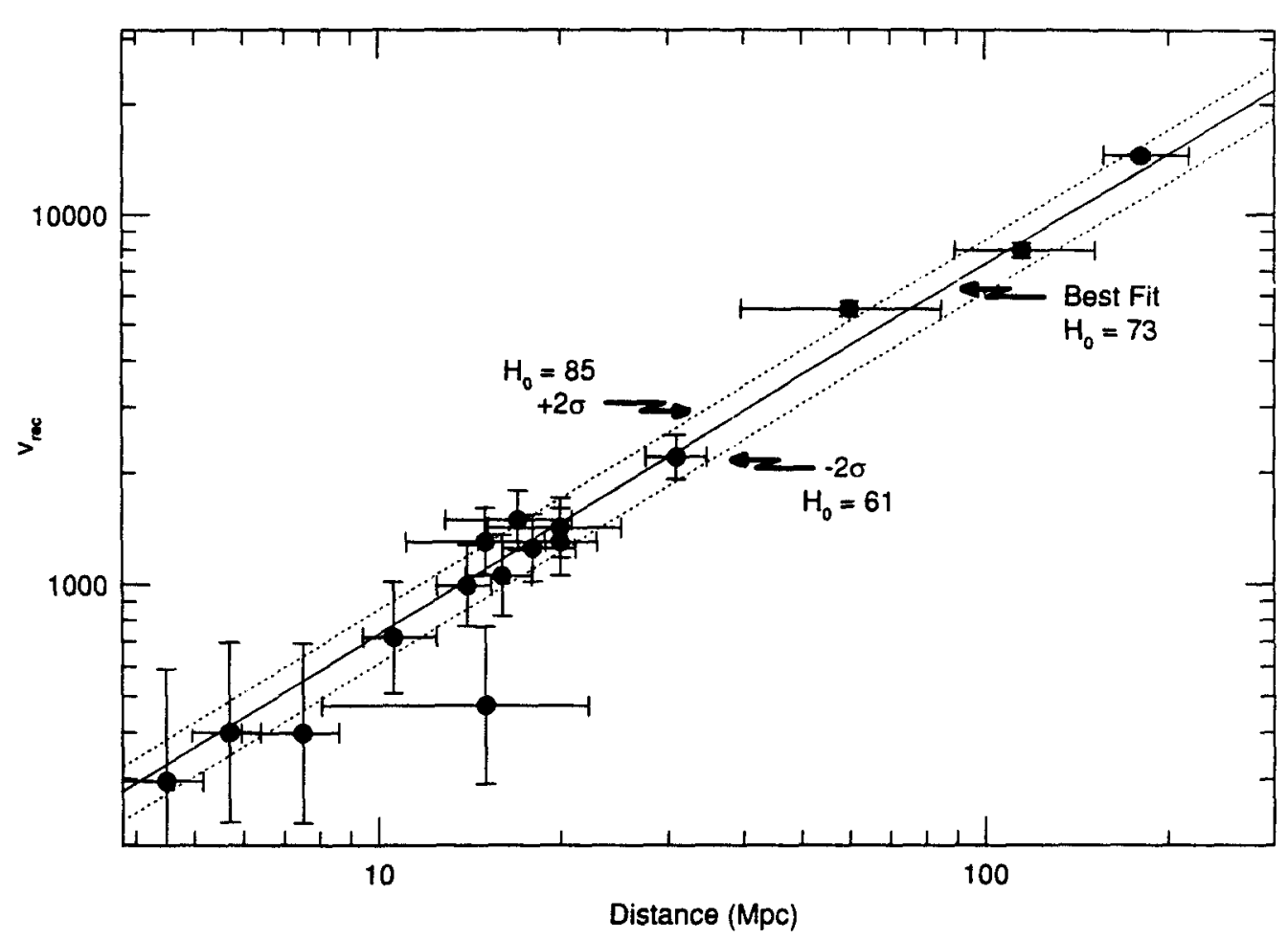

FIGURE 3. Hubble diagram based on EPM distances to SNII.

near zero or negative, and we exclude these objects in our determination of $H_{0}$. Using the EPM distances as shown in Figure 3, and assigning $300 \mathrm{~km} \mathrm{~s}^{-1}$ errors (representing uncertainties due to large scale peculiar motions not associated with Virgo infall) to all recession velocities, we derive a value of $H_{0}=73 \pm 6$ (statistical) $\mathrm{km} \mathrm{s}^{-1} \mathrm{Mpc}^{-1}$, using the least-squares technique for data sets with errors in both directions described by Press et al. (1992). The value of $H_{0}$ derived from our sample depends somewhat on the velocity errors assigned to each galaxy, ranging from $H_{0}=70 \pm 3 \mathrm{~km} \mathrm{~s}^{-1} \mathrm{Mpc}^{-1}$, if velocity errors are assumed to be zero, to $H_{0}=75 \pm 7 \mathrm{~km} \mathrm{~s}^{-1} \mathrm{Mpc}^{-1}$ if we assign errors of $600 \mathrm{~km} \mathrm{~s}^{-1}$ to each velocity measurement. The nearby galaxies in our sample give a smaller value of $H_{0}$ than those further away, and their weight depends on the error adopted. It is difficult to assign a realistic overall error to our value of $H_{0}$ because the statistical uncertainty of the measurement $(8 \%)$ is of the same order as possible systematic uncertainties in EPM.

The Expanding Photosphere Method is subject to errors due to uncertainties in our understanding of SNe II physics. All of the distances used in this determination of $H_{0}$ are based on the atmospheric code developed by Eastman (Eastman \& Kirshner 1989; Eastman \& Pinto 1993). It may be possible to evaluate the accuracy of EPM by comparing the output of other atmospheric codes which make slightly different physical assumptions in computing model atmospheres. A limited comparison can be made in the case of SN 1987A, a SN which several groups modeled (Eastman \& Kirshner 1989; Höflich 1988; Schmutz et al. 1990; Chilkuri and Wagoner 1988), where the agreement 
is better than $\pm 10 \%$. Assigning an underlying uncertainty of $\pm 10 \%$ to EPM, we find $H_{0}=73 \pm 6$ (statistical) \pm 7 (systematic) $\mathrm{km} \mathrm{s}^{-1} \mathrm{Mpc}^{-1}$.

The importance of SN searches to this program cannot be overstated. None of the five objects presented here was discovered accidentally: all were found as the result of systematic searches of galaxies for supernovae. As these searches continue, they will enable us to apply EPM to many SNe II at redshifts larger than $c z=5000 \mathrm{~km} \mathrm{~s}^{-1}$. These objects, which will typically be between $17<m_{v}<20 \mathrm{mag}$, combined with those already measured, will allow us to derive a value of $H_{0}$ which is robust against large-scale perturbations in the Hubble flow. In addition, there is no technical reason why we cannot apply EPM to SNe II discovered at redshifts beyond $z=0.1$, giving us the possibility of a direct approach to measuring the deceleration parameter, $q_{0}$.

\section{Acknowledgements}

Many people do the work: just one gives the talk. I am grateful for the contributions of Brian Schmidt and Ron Eastman to this effort. Supernova research at the Harvard University is supported by NSF grant AST 92-18475 and NASA grants NAG 5-841, NGT-51002, and grant GO-2563.01-87A through the STScI.

\section{REFERENCES}

Aaronson, M. et al. 1982, ApJS, 50, 241

Baron, E., et al. 1993, ApJ, 416, L21

Branch, D., Falk, S. W., McCall, M. L., Rybski, P., Uomoto, A. K., \& Wills, B. J. 1983, ApJ, 244,780 .

Branch, D. \& Miller,D.L. 1993, ApJ, 405, L5

Chilkuri, M. \& Wagoner, R. V. 1988, in Atmospheric Diagnostics of Stellar Evolution, IAU Colloquium 108, ed. K. Nomoto, (Berlin: Springer-Verlag) p295

Cook, K. H., Aaronson, M., \& Illingworth, G. 1986, ApJ, 301, L45

Eastman, R. G. \& Kirshner, R. P. 1989, ApJ, 347, 771

Eastman, R. G., Schmidt, B. P., \& Kirshner, R. P. 1994, ApJ, submitted

Eastman, R. G. \& Pinto, P. A. 1993 ApJ, 412, 731

Fransson et al. 1989, ApJ, 336, 429

Freedman, W. L. et al. 1994, ApJ, 430, 53

Gould, A. 1994, ApJ, 425, 51

Höflich, P. 1988, in Atmospheric Diagnostics of Stellar Evolution, IAU Colloquium 108, ed. K. Nomoto, (Berlin: Springer-Verlag) p288

Hamuy, M. et al. 1993, AJ, 106, 2392

Huchra, J. P. 1988, in Proceedings of the A. S. P.: The Extragalactic Distance Scale, ed. S. van den Bergh \& C. J. Pritchet, (Provo: Brigham Young University Press) p 257

Jakobsen, P. et al. 1991, ApJ, 369, L63

Jacoby, G. H., Branch, D., Ciardullo, R., Harris, W. E., Pierce, M. J., Pritchet, C. J., Tonry, J. L., \& Welch, D. L. 1992, PASP, 104, 599

Januzzi, B., Schmidt, G., Elston, R. \& Smith, P. 1993, IAU Circular \#5776

Kirshner, R. P. 1985, in Lecture Notes in Physics: Supernovae as Distance Indicators, ed. N. Bartel (Berlin: Springer-Verlag), p. 171

Kirshner, R. P. \& Kwan, J. 1974, ApJ, 193, 27

Kirshner, R.P., Winkler, P.F., \& Chevalier, R.A. 1987, ApJ, 315, L135.

Lauer, T. L. \& Postman, M. 1992, ApJ, 400, L47 
Lauer, T. R. \& Postman, M. 1993, in Texas/PASCOS 92: Relativistic Astrophysics and Particle Cosmology ed. C. W. Akerlof \& M. A. Srednicki (Ann. N.Y. Acad. Sci) 688, 531

Leibundgut, B. et al. 1993, AJ, 105310

Lynden-Bell, D., Faber, S. M., Burstein, D., Davies, R. L., Dressler, A., Terlevich, R. J., \& Wegner, G. 1988, ApJ, 326, 19

Mathewson, D. S., Ford, V. L., \& Buchhorn, M. 1992, ApJ, 389, L5

McCall, M. 1993, ApJ, 417, L75

Phillips, M.M. et al. 1992, AJ, 103, 1632

Phillips, M. M. et al. 1993, ApJ, 413, L105

Pierce, M. J. 1994, ApJ, 430, 53

Plait, P.C., et al. 1994, Ap.J. submitted

Press, W. H., Flannery, B. P., Teukolsky, S. A., \& Vettering, W. T. 1992. Numerical Recipes in C, 2nd ed. (Cambridge University Press: Cambridge)

Pun, C.C., Kirshner, R.P., \& Sonneborn, G. 1994 in preparation

Renzini, A. 1991. in Observational Tests of Cosmological Inflation, ed. T. Shanks et al. (Dordecht: Kluwer), p 313

Ruiz-Lapuente, P. et al. 1993, Nature, 365, 728

Sandage, A. 1994, ApJ, submitted

Sandage, A. \& Tammann, G. A. 1990 ApJ, 365, 1

Sandage, A. \& Tammann, G. A. 1993 ApJ, 415, 1

Sandage, A., Saha, A., Tammann, G. A., Panagia, N., \& Machetto, F. D. 1992, ApJ, 401, L7

Sandage, A., Saha, A., Tammann, G. A., Labhardt, L., Schwengeler, H., Panagia, N., \& Machetto, F. D. 1994, ApJL, submitted.

Schechter, P. L. 1980, AJ, 85, 801

Schmidt, B. P., Kirshner, R. P., \& Eastman, R. G. 1992, ApJ, 395, 366

Schmidt, B. P., Kirshner, R. P., \& Eastman, R. G., Grashuis, R., Dell'Antonio, I., Caldwell, N., Foltz, C., Huchra, J., \& Milone, A. A. E., 1993, Nature, 364, 600

Schmidt, B. P., Kirshner, R. P., Eastman, R. G., Phillips, M. M., Suntzeff, N. B., Hamuy, M., Aviles, R., Filippenko, A. V., Ho, L., Matheson, T., Grashuis, R., Maza, J., Kirkpatrick, J. D., Kuijken, K., Zucker, D., Bolte, M., \& Tyson, N. 1994a, AJ, 107, 1444

Schmidt, B. P. et al. 1994b, ApJ, 432, 42

Schmutz, W., Abbot, D. C., Russell, R. S., Hamann, W. R., \& Wessolowski, U. 1990, ApJ, 355, 255

Smith, R.C., Kirshner, R.P., Blair, W.P., \& Winkler, P.F 1991, ApJ, 375, 652

Tully, R. B. \& Fisher J. R. 1977 A\&A, 54, 661

Wagoner, R. V. 1977, ApJ, 214, L7

Wagoner, R. V. 1981, ApJ, 250, L65

Walker, A. R. 1987, MNRAS, 225, 627 